\title{
La gestion du risque barrage - vallée du Portugal
}

\author{
Dam-valley risk management in Portugal \\ par Antonio Betâmio de Almeida \\ Université Technique de Lisbonne \\ (IST - CEHIDRO)
}

After a review of the risk concept within the present society framework, as well as the technology knowledge and public perception, that concept is applied to valleys with dams. It is also presented the main concepts related to risk management, valley vulnerability and computational systems developped in Portugal by a NATO research project. The benefits obtained by this project are referred specially in what concerns the civil protection level and the valley safety procedures.

\section{I $\square$ LA SÉCURITÉ ET LE RISQUE DANS LES VALLÉES AVEC DES BARRAGES}

\subsection{Introduction}

Les barrages et les réservoirs d'eau créés dans les vallées jouent un rôle très important dans le cadre de la gestion de l'eau et apportent une contribution à la qualité de vie de la société. Les barrages constituent également un danger potentiel pour les vallées à l'aval. En effet, quelqu'en soit la cause et la probabilité d'occurrence, la faillite de la structure en ce que concerne sa capacité à retenir l'eau est toujours possible. Les barrages suscitent souvent l'appréhension de l'opinion publique quant aux impacts sur l'environnement, notamment le risque de crue potentielle provoquée à l'aval par une rupture structurelle. Les modifications des conditions hydrologiques et le vieillissement des barrages sont aussi des facteurs à prendre en compte. Les bénéfices associés au progrès technologique résultant des grands ouvrages sont, dans la période contemporaine, moins évidents et sont, parfois, soumis à une critique féroce. Il y a des raisons pour cette sensibilité accrue : la société actuelle devient plus vulnérable aux risques naturels et technologiques, et la société ouverte et globalisée, encadrée par les media, constitue un nouveau pouvoir et une contrainte considérable par rapport à la prise de décision (société de risque). L'opinion et la participation du public seront de plus en plus des facteurs majeurs à intégrer.

Les règlements et recommandations actuels concernant la sécurité des barrages incluent des consignes très strictes en ce qui concerne la sécurité à l'aval. Dans ce contexte, on a conclu qu'il était nécessaire de renouveler les concepts, les moyens et le discours sur le risque des barrages et des vallées à l'aval face aux complexités inhérentes à ce paradigme. Afin d'aboutir à une méthodologie de gestion du risque plus poussée et intégrée, le "Laboratorio nacional de Engenharia Civil" (LNEC) et l'Université technique de Lisbonne (ISTCEHIDRO) ont proposé, en 1992, un projet de développement à I'OTAN dans le cadre du "Science for Stability Program". Le projet a démarré en 1994 et est en train de se terminer (2000). Le projet a également eu le soutien de l'Autorité Portuguaise pour l'Eau et la Sécurité des Barrages (INAG), du Service National de Protection Civile (SNPC) et d'"Electricidade de Portugal" (EDP). En ce qui concerne l'objet de cet article, le projet a, entre autres, les objectifs principaux suivants : 1) améliorer les logiciels pour la simulation des crues provoquées par la rupture de barrages, notamment le développement de modèles hydrodynamiques avancés et validés le mieux possible; 2) introduire les méthodologies des sciences sociales appliquées à la gestion du risque, en particulier en ce qui concerne la perception du risque par le public, la communication et l'aménagement de la vallée pendant la vie du barrage; 3 ) développer des logiciels pour aider à la gestion du risque des barrages et des vallées, basés sur les nouvelles technologies de l'information (e.g. SIG et bases de données multimedia).

Certains des concepts et des outils développés dans le cadre du projet sont maintenant en train d'être appliqués au processus de gestion intégrée du risque contre les crues exceptionnelles et naturelles dans les vallées portugaises. 


\subsection{Sécurité et risque dans les vallées comportant des barrages}

Les experts et les citoyens ont, en général, une perception différente de l'incrément et de la signification du risque dû aux barrages. Entre la confiance du spécialiste ou la foi dans le pouvoir technologique, et la suspicion et la peur, liées aux incertitudes d'une modification réalisée par l'homme, on peut trouver un spectre varié de réactions. Dans la société contemporaine, la perception du risque est en train de constituer un facteur de changement des conditions de l'exercice du pouvoir et de la décision, et de briser beaucoup des barrières traditionnelles entre les scientifiques, les autorités et le public. En ce qui concerne les vallées, la sécurité ne peut pas être confinée à la surveillance des conditions de l'état structurel et d'exploitation des barrages. Pour faire face à la complexité du problème, on doit alors considérer deux concepts de base :

- la gestion intégrée du risque et de la sécurité, intégrant, dans leur ensemble, les sous-systèmes physiques barrages (et les réservoirs) et vallée à l'aval et le sous-système social et économique relatif à la vallée (Figure 1) ;

- le partage de la responsabilité et de la gestion du risque, négocié entre tous les acteurs en prenant en considération les perceptions et les sentiments humains vis-à-vis de la définition du niveau de risque accepté et de l'évolution future des conditions de sécurité et de vulnérabilité de la vallée (Figure 2).

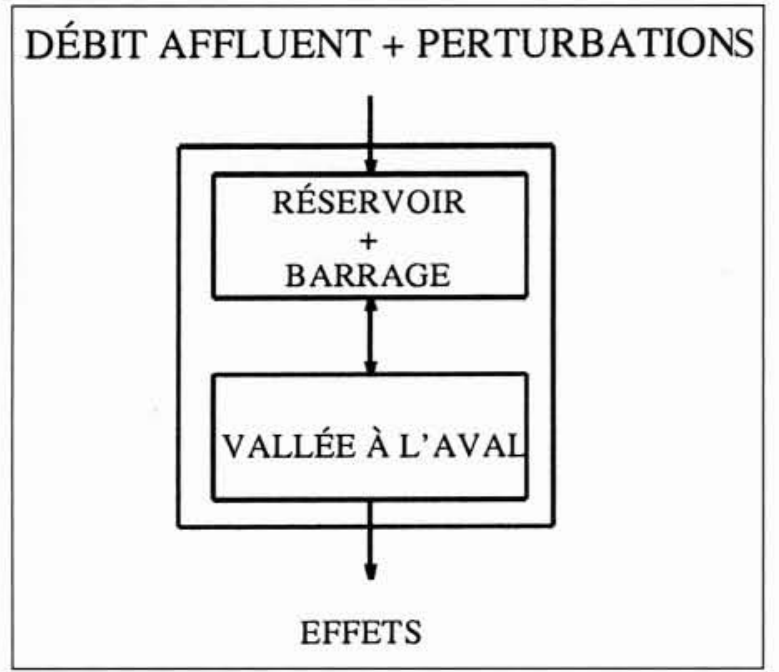

1. Système intégré du barrage et de la vallée à l'aval.

Dans le cadre de ces deux concepts, on doit inclure également le contrôle des crues naturelles, les débits déversés par les barrages et les dangers hydro-géologiques spécifiques à chaque vallée. Ces concepts peuvent aussi être adaptés au cas des digues de protection contre les inondations.

Dans un cadre conceptuel, le soussystème barrage-réservoir répond à chaque perturbation externe ou interne. Cette réponse peut induire un danger potentiel pour le système vallée à l'aval. Les vecteurs de ce danger sont les crues provenant des barrages, et les pertes potentielles, humaines, écono-

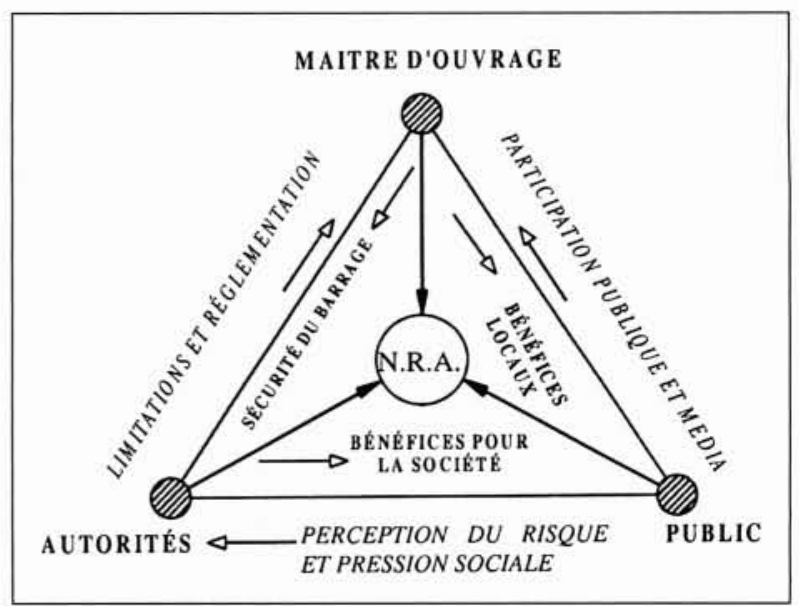

2. Partage de responsabilité dans la définition du niveau de risque accepté à l'aval (N.R.A.).

miques ou de l'environnement, sont le résultat de la vulnérabilité de la vallée vis-à-vis des crues. Pour chaque événement potentiellement dangereux, Ei pour le système barrageréservoir, on peut considérer que la définition formelle du risque intégré (Figure 3 ), Ri, est composée par le risque interne (barrage) et le risque externe (vallée à l'aval). Dans cette définition, $\mathrm{P}(\mathrm{Ei})=$ probabilité d'occurrence de l'événement, $\mathrm{P}(\mathrm{Qp} / \mathrm{Ei})=$ probabilité conditionnelle d'occurrence d'une crue exceptionnelle de débit de pointe Qp provoquée par la réponse du barrage à l'événement $\mathrm{Ei}$ et $\mathrm{P}$ $(\mathrm{N} / \mathrm{Qp})=$ probabilité conditionnelle de l'occurrence de pertes globales (e.g. nombre de victimes) dues à la crue avec le débit de pointe Qp provoqué par la réponse du barrage.

Les consignes de sécurité du barrage, pendant les phases de la vie du projet, construction, exploitation et abandon, contribueront à la réduction du risque interne et les actions pour la sécurité des vallées contribueront à la réduction du risque externe dû aux crues. Les pertes à l'aval dues à une crue dépendent de l'intensité ou de l'agressivité de celle-ci et de la fragilité de la vallée. Le concept de vulnérabilité a pour but de donner une signification opérationnelle à la résistance de la vallée et, d'une façon indirecte, à la probabilité $\mathrm{p}(\mathrm{N} / \mathrm{Qp})$. Contrôler ou limiter la vulnérabilité de la vallée, c'est aussi contrôler la sécurité et le risque induits par les crues et les conséquences d'un événement dangereux sur un barrage. Les pertes à l'aval seront fonction de la réponse d'ensemble du barrage, de la vallée (y compris ses habitants) et des dispositifs de protection civile. La gestion intégrée du risque et de la sécurité barrage-vallée s'impose alors naturellement.

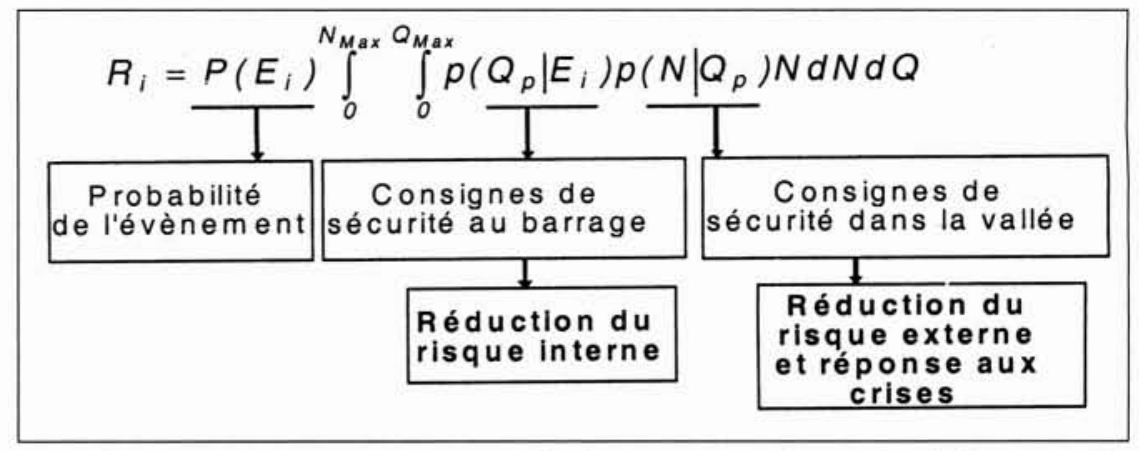

3. Définition formelle du risque intégré (barrage-vallée) dû à l'événement Ei. 


\subsection{Prévention intégrée du risque}

La gestion du risque barrage-vallée peut être considérée comme composée de deux parties : le processus d'évaluation du risque, ayant pour but la quantification du risque, et le processus de prévention intégrée du risque (Figure 4) ayant pour but le contrôle du risque dans la vallée. Cette prévention a deux volets : le contrôle et la réduction du risque et de la vulnérabilité, et la réponse aux situations de crise dues aux crues dans la vallée.

Le premier volet contient toutes les actions actives ou passives pour réduire les probabilités de réponse présentées sur la Figure 3. Ces actions incluent les opérations et consignes de sécurité pour chaque barrage et vallée. Le second contient les actions à entreprendre en cas d'incident et de crue catastrophique (actions de protection civile, d'assistance d'urgente, d'évacuation et post-urgence). Il ne faut pas oublier que dans les vallées à l'aval des barrages, le concept de défense structurelle n'est plus valable et des alternatives non-structurelles doivent être trouvées. Dans la gestion de ce type de risque, on doit alors suivre les stratégies suivantes : 1) Le principe de prévention des accidents, qui a trait à la réduction du risque interne, à la charge de l'exploitant du barrage; 2) Le principe de mitigation des dommages, qui a trait à la réduction du risque externe, à la charge des gestionnaires de la vallée. Le partage de responsabilité ne signifie pas un affaiblissement de celle-ci mais, par contre, un incrément dans les responsabilités respectives, complémentaires et plus solides, dans le contexte de la société de risque contemporaine. La prévention effective des risques associés aux accidents potentiels de barrage et l'amélioration de la sécurité dans les vallées sont devenues une préoccupation dans beaucoup pays, y compris le Portugal. En effet, le Règlement de Sécurité des Barrages Portugais (1990) oblige le maître d'ouvrage à présenter des études de propagation de l'onde de crue provoquée par des scénarios de rupture de barrage et la préparation de plans d'urgence et d'évacuation à l'aval, y compris les systèmes d'alarme. La complexité de l'application de ce type de règlement est très grande : l'agressivité des crues déclenchées par une rupture d'un barrage ou d'une série de barrages (rupture en domino) vis-àvis de l'extrême fragilité des vallées très peuplées, sans aucune préparation et perception positive des risques et des mesures de protection, pose des difficultés très sérieuses et rompt avec les attitudes traditionnelles des pouvoirs publics et des responsabilités individuelles et collectives.

Le succès d'une prévention intégrée du risque est lié au développement de nouvelles méthodologies : 1) modélisation sur ordinateur des scénarios de rupture et de propagation des crues ; 2) introduction des sciences sociales dans le processus de contrôle de sécurité des vallées ; 3 ) évaluation de la vulnérabilité de la vallée et des dommages et pertes potentiels, ayant pour base les plans d'inondation, les caractéristiques de l'occupation socio-économique, la perception du risque par le public et le système de réponse en cas de crise; 4) développement de systèmes d'information automatiques pour l'aide à la décision et à la gestion du risque et des situations de crise, basés sur un SIG et des données de base spéciales; 5) développement des plans d'urgence avec la participation des élus locaux et des recommandations sur l'aménagement et l'occupation de la vallée.

Les méthodologies développées dans le cadre du projet OTAN FLOODRISK sont en train d'être appliquées à un cas d'étude : la vallée de l'Arade au sud du Portugal (Algarve), avec deux barrages (barrages du Funcho et du Arade) et deux villes à l'aval (Sines et Portimão). Un modèle physique (échelle 1/150) de la vallée a été aussi construit au LNEC. Le prototype du plan d'urgence servira de modèle pour les autres plans à préparer pour toutes les vallées à l'aval des barrages de plus de $15 \mathrm{~m}$ de hauteur ou ayant un réservoir de volume supérieur à $100000 \mathrm{~m}^{3}$ (environ un millier de barrages sous le régime du règlement portugais). Des résultats partiels du projet sont présentés de [1] à [5].

\section{II — PLANS D'URGENCE ET PERCEPTION SOCIALE}

\section{- 2.1 Plans d'urgence pour les vallées}

Les plans d'urgence sont des outils fondamentaux pour la protection de la vallée à l'aval des barrages et pour atténuer les dommages provoqués par les crues. Pour atteindre ce désiderata, la préparation de ce type de plan doit inclure quatre volets: 1) l'évaluation des caractéristiques probables des crues et les effets (pertes et dommages) de celles-ci à l'aval des barrages; 2) la préparation des actions pour réduire l'impact des crues associées aux ruptures de barrages, y compris la préparation des mesures, passives (contrôle de l'occupation du sol) et actives (systèmes d'alarme, plans d'évacuation, information et préparation du public et des autorités locales); 3) la préparation de la réponse adéquate de la protection civile et des populations à un incident ou une situation de crise; 4) la préparation de moyens de sauvetage et d'appui à la vallée. Dans le cadre des concepts déjà présentés, deux classes de plans d'urgence peuvent être envisagées : 1) le plan d'urgence interne, relatif aux actions de sécurité spécifiques à chaque barrage; 2) le plan d'urgence externe, relatif aux actions de protection dans la vallée à l'aval de chaque barrage.

Le plan d'urgence interne doit inclure: 1) les caractéristiques du barrage et des structures de contrôle de 
l'eau; 2) l'identification des situations potentiellement dangereuses pour le barrage; 3 ) la définition des différents niveaux de sécurité et d'urgence concernant l'exploitation du barrage; 4) les méthodologies pour la détection des événements dangereux pour le barrage; 5) les règles d'alerte des autorités de sécurité des barrages et des autorités locales; 6) les règles d'alerte et de mobilisation des services de protection civile.

Le plan d'urgence externe doit inclure: 1) les caractéristiques les plus importantes de la vallée à l'aval, notamment l'occupation humaine et économique, les installations stratégiques du point de vue social; 2) la définition des systèmes d'alerte (alarme) et d'évacuation des habitants en danger; 3) l'identification des responsables de la sécurité et la définition de leurs responsabilités et fonctions respectives; 4) la localisation des ressources de secours; 5) l'identification des zones protégées et les chemins d'accès; 6) l'identification des systèmes de communication et de transport disponibles en cas d'urgence.

\subsection{La perception sociale et la participation du public}

La gestion effective du risque et la préparation des plans d'urgence demandent, dans le monde contemporain, une rupture avec des idées acquises sur la gestion des désastres et le comportement humain sous les effets des événements exceptionnels. L'ampleur et l'impact des risques physiques dépendent, dans une communauté donnée, des facteurs suivants : 1) le système culturel et de valeurs et la perception du risque; 2) la façon dont les organisations et les décideurs gèrent les vulnérabilités régionales et locales; 3) le niveau de préparation avant le désastre et la capacité de gestion en cas de crise. La gestion du risque dans les vallées doit se baser sur des attitudes pro-actives et sur la conviction qu'on peut faire beaucoup pour réduire les pertes éventuelles. Dans ce cadre, les populations et les autorités locales ont un rôle important. Elles peuvent et doivent participer à des actions de prévention et de préparation afin d'assurer un comportement adéquat en cas de crise et de respecter le NRA dans chaque vallée (Figure 2) par une politique cohérente de l'occupation du sol vis-à-vis du risque dû aux crues. A notre avis, considérer les plans et les risques d'inondation comme des informations réservées et ne pas considérer ces risques dans la gestion et l'occupation du sol de la vallée est contraire au contrôle de la sécurité et à la réduction de la vulnérabilité.

Dans le cadre du projet OTAN on a fait, pour la première fois au Portugal, des études de terrain sur la perception du risque des populations à l'aval des barrages $[2,3]$ : une première étude exploratoire avec des interviews des résidents, élus et autorités locaux et une deuxième étude basée sur un questionnaire structuré fourni à un échantillon représentatif de la population exposée au risque. De cette étude on a retiré, parmi d'autres, les conclusions générales suivantes :

- il y a une surestimation des bénéfices personnels, locaux et régionaux des barrages, et une sous-estimation des risques dus à ceux-ci, mais on a découvert des "illusions positives" dans les perceptions des populations qui habitent juste à l'aval des barrages;

- la perception du risque semble être affaiblie et la mise en place des mesures de protection contre les crues et accidents doit être activée. Après la construction des barrages, les crues naturelles sont perçues, dans la vallée étudiée, d'une façon moins dramatique avec une sensation de fausse sécurité;
- la perception du risque et des bénéfices décroît avec la distance séparant les individus et le barrage. Il y a également une différence entre les perceptions des populations urbaines et rurales.

\section{VULNÉRABILITÉ DES VALLÉES}

Pour être efficace dans le contrôle du risque externe, le concept de vulnérabilité doit être structuré pour en permettre la quantification. Pour aboutir à cet objectif, un index de vulnérabilité a été défini, basé sur deux composants [2,5] :

- L'index d'agressivité, fonction des caractéristiques physiques de la crue provoquée par la rupture potentielle du barrage, estimé en chaque zone de la vallée.

- L'index de fragilité, fonction des caractéristiques sociales, économiques et environnementales de la vallée et de la capacité de résistance et de protection contre les inondations, le long de chaque zone de la vallée à l'aval.

L'index d'agressivité est fonction des caractéristiques hydrodynamiques des crues. L'index de fragilité est composé par une somme de termes qui caractérisent les aspects suivants : 1) vulnérabilité humaine, fonction du nombre d'habitants exposés au risque, du système d'alerte, du plan d'urgence et d'évacuation, de la zone inondable et des caractéristiques sociologiques de la population; 2) la vulnérabilité structurelle des bâtiments et des infrastructures stratégiques; 3) la vulnérabilité de l'environnement; et 4) la vulnérabilité économique, fonction du type d'occupation économique de chaque zone. Un algorithme préliminaire a été développé pour quantifier cet index [5]. L'expérience et l'analyse systématique permettront la calibration du modèle d'évaluation de cet index. Celui-ci pourra, du point de vue théorique, être la base du contrôle de la maîtrise du risque externe par les mesures et les actions de prévention dans la vallée.

\section{IV — LOGICIELS D'APPUI A LA GESTION DU RISQUE}

La gestion intégrée du risque doit faire face à des processus complexes et l'appui des logiciels est indispensable pour assurer le succès effectif du contrôle de la vulnérabilité. Afin de faciliter les phases de prévention des accidents, on a développé dans le cadre du projet OTAN un système d'appui (Dam Support System) constitué par (Figure 5) : un soussystème d'information (Daminfo), composé d'une base de données relationnelle, d'un Système d'Information Géographique et d'une interface pour l'accès à ces deux ensembles

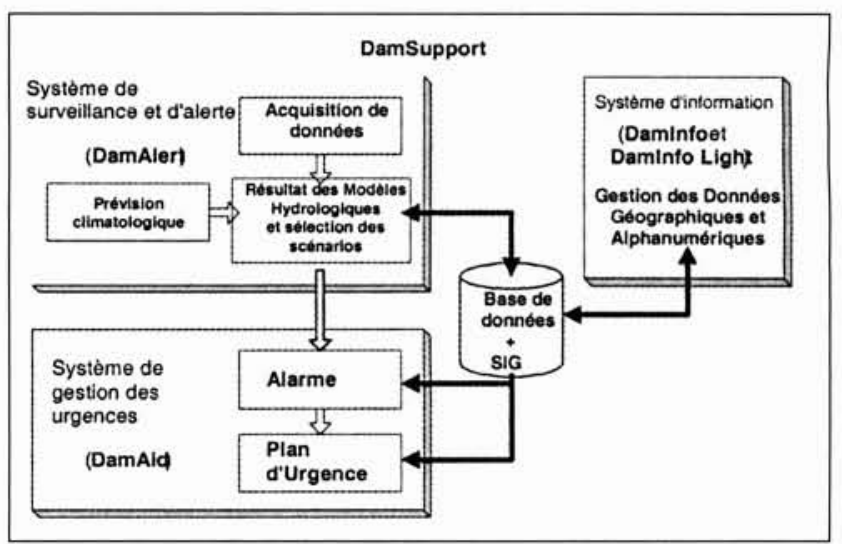

5. Les principaux composants du Système Dam Support. 
de données ; un ensemble de modules (Dam Alert) pour contrôler, simuler ou prévoir des événements hydrologiques et hydrauliques et aussi pour déclencher l'alarme en situation de danger potentiel; et un sous-système d'appui direct à la gestion en situation d'urgence ou de crise (Dam Aid) en ce qui concerne l'alerte aux populations, l'alerte aux autorités et l'activation des plans d'urgence externes et d'aide aux habitants en zone de risque. Ces sous-systèmes dépendent d'un système de communications robuste et efficace entre les acteurs concernés : maîtres d'ouvrage, autorités de sûreté des barrages, services de protection civile, autorités locales, pompiers, forces armées, entre autres.

\section{CONCLUSIONS}

Le risque généré par un barrage pour l'environnement, et en particulier la sécurité dans la vallée à l'aval, est un problème très complexe ; la gestion intégrée et le partage du risque deviennent de plus en plus indispensables. La définition du concept de vulnérabilité pourra fournir une aide pour le contrôle de l'efficacité des mesures de protection et la caractérisation de chaque vallée par rapport au risque crues. La sécurité peut être améliorée grâce à l'aménagement intégré du territoire (restrictions dans l'occupation du sol) et l'étude de la perception du risque pour encadrer les actions d'information et de participation du public. L'importance des sciences sociales dans les études sur le risque est une conséquence d'un principe de base : les risques constituent un objet d'étude et d'analyse uniquement dans la mesure où ils se produisent dans le cadre du système social et sont également une construction sociale. Les sciences sociales peuvent être associées aux systèmes opérationnels de la protection civile, en particulier les réseaux d'alerte. Les outils informatiques intégrés, apportant l'appui à la décision dans le domaine de la gestion du risque, constituent une contribution pour la sécurité des vallées à l'aval des barrages.
Au Portugal, les retombées du projet OTAN sont en train de devenir une réalité offrant une sécurité accrue dans les vallées vis-à-vis des crues exceptionnelles, grâce à de nouvelles mesures (plans d'urgence) et des méthodologies plus intégrées.

\section{ÉQUIPE DU PROJET}

Les principaux responsables du projet OTAN sont les suivants : A. Betâmio de Almeida (Directeur), C. Matias Ramos (Sous-Directeur), Alzira Santos, Bento Franco. Teresa Viseu, Delta Silva et Miguel Gamboa. Le Professeur Erich Plate est l'expert de I'OTAN pour le projet. L'auteur remercie l'effort fourni par tous les collaborateurs du projet.

\section{Bibliographie}

II] ALMEIDA. A. B. et al. 2000. Dam-Valley Risk Management. First Results of a Case Study in Portugal. Arade Valley. Proceedings 20th ICOLD Congress (à paraître), Beijing, China.

[2] ALMEIDA, A. B \& VISEU, T. 1997. Dams and Safety Management a Downstream Valleys (eds), Rotterdam. Balkema.

[3] ALMEIDA, A. B. et al. 1997. Dam-Break Flood Risk and Safety Management at Downstream Valleys : a Portuguese Integrated Research Projet. Proceedings 19th ICOLD Congress, Florence, Italy, Vol IV, (Q. 75 R. 25): $331-347$.

[4] LIMA. M. L. et al. 1997. From Risk Analysis to Risk Perception Developing a Risk Communication Strategy for a Dam-Break Flood Risk. In C. Guedes Soares (ed.), Advances in Safety and Reliability, Vol. 1, Oxford, Pergamon: 53-60.

[5] ALMEIDA. A. B. 2000. Flood Plain Dam Risk Management Downstream Dams. A Research Projet and a Case Study. In New Trends in Water and Environmental Engineering for Safety and Life : Eco-compatible Solutions for Aquatic Environments, Rotterdam, Balkema. 